\title{
Artículo Especial: Guía de lectura crítica de estudios observacionales en epidemiología (primera parte)
}

\author{
Critical appraisal guide of observational studies in epidemiology (first part)
}

Agustín Ciapponi ${ }^{\star}$

\begin{abstract}
Resumen
Se describen en este artículo la estructura general de los diferentes tipos de estudios observacionales utilizados en epidemiología, su diseño, sus ventajas y desventajas, ilustrándolos con ejemplos. Se describen las series de casos y los estudios de corte transversal. Al final del artículo se adjunta una lista de cotejo para la crítica la lectura de este tipo de estudios observacionales.

En una segunda entrega de EVIDENCIA se abordarán aspectos de la lectura crítica de los estudios de casos y controles, los estudios de cohorte y los estudios "ecológicos" o de correlación.

Abstract

The general structure of different types, of observational studies used in epidemiology, their design, their advantages and their disadvantages are described in this article. The concepts are illustrated with examples. Case series and cross sectional studies are described. At the end of the article a checklist for critical appraisal is provided.

In a second article of EVIDENCIA will be discussed some aspects of critical appraisal of case control studies, cohort studies, and "ecologic" (or correlation) studies.
\end{abstract}

Palabras clave: epidemiología, estudios de cohortes, estudios de casos y controles, estudios transversales, series de casos, lectura crítica. Key words: epidemiology, cohort studies, case control studies, cross sectional studies, case series, critical appraisal.

Ciapponi A. Guía de lectura crítica de estudios observacionales en epidemiología. Evid. actual. práct. ambul; 13(1): 135-140. Oct-Dic 2010.

\section{Introducción}

Clásicamente la epidemiología es definida como el estudio de la distribución y de los determinantes de las enfermedades o problemas de salud en una población específica, y la aplicación de este estudio al control de los problemas de salud . Por otro lado, la epidemiología clínica es definida como la aplicación de los conocimientos, la metodología y el razonamiento epidemiológicos a los problemas de la medicina clínica ${ }^{2}$, con el objetivo de ayudar al médico a resolver los problemas de salud de sus pacientes, interpretar la información clínica y mejorar la calidad de su investigación'.

Los estudios epidemiológicos han servido para conocer las causas, y también intervenciones preventivas y terapéuticas de las enfermedades transmisibles durante la primera mitad del siglo XX, y en adelante, de las enfermedades crónicas como el cáncer y las enfermedades cardiovasculares.

La epidemiología se utiliza para describir el estado de salud/enfermedad de la población. Para las autoridades de la salud pública es muy importante conocer la carga de enfermedad en la población, con el objetivo de poder planificar, priorizar y asignar recursos. Además, la epidemiología se está utilizando para evaluar la efectividad y la eficiencia de los servicios sanitarios, desde la efectividad de un programa de rastreo o de un determinado tratamiento o bien, el impacto de una determinada política social.

Por siglos, los estudios observacionales fueron la base de investigación clínica. Los clínicos desde la época de Hipócrates describieron las enfermedades de sus pacientes, las presentaciones y sus esfuerzos terapéuticos. Los ejemplos son innumerables. Leonardo da Vinci observó los exámenes postmortem con el propósito de describir la anatomía humana a través de sus dibujos. Los comentarios de Harvey ayudaron explicar la naturaleza de la circulación. Aún con el surgimiento de los ensayos clínicos, la investigación clínica dependía de estudios observacionales de describir la salud, la enfermedad y patrones asociados ${ }^{3}$.

Para responder a estas preguntas, la epidemiología ha desarrollado una metodología propia, basada más en la observación que en la experimentación, puesto que no sería ético "experimentar" con poblaciones, por ejemplo asignándole una potencial exposición cancerígena a un grupo de personas, y a otro no, para evaluar la incidencia de un tipo de cáncer. Los diseños epidemiológicos definen, por lo tanto, estudios observacionales (o no experimentales) basados en el tipo de muestreo que se hace de las poblaciones.

El tipo de diseño epidemiológico responderá, por lo tanto, a la pregunta o a las preguntas que los investigadores se hagan de la población y de la condición (enfermedad, factor de riesgo o preventivo, actividad asistencial, etc.) a estudiar ${ }^{4}$.

La epidemiología emplea muchos de los procedimientos de las ciencias sociales, se basa en el análisis estadístico, y configura una metodología propia y específica que la caracteriza como disciplina científica. Sin embargo, los métodos de la epidemiología y, más concretamente, sus limitaciones como ciencia básica para la investigación etiológica y sus implicaciones para la salud pública hacen imprescindible una detallada evaluación crítica de la evidencia que provee ${ }^{5}$

Presentaremos en este artículo, listas de cotejo de lectura crítica para los principales diseños observacionales.

La epidemiología también puede denominarse descriptiva si sólo pretende describir la ocurrencia de un evento (reportes y series de casos, estudios ecológicos) o analítica si se pretende investigar las causas o factores asociados a un evento determinado (estudios de corte transversal, caso-control, y cohortes).

La toma de decisiones se basa, entre otras cosas, en los resultados de los diferentes estudios, o en las conclusiones de las Revisiones sistemáticas o los meta-análisis que los incluyen. Por tanto es necesario disponer de instrumentos que evalúen y permitan calificar y clasificar de forma adecuada la evidencia disponible. Los instrumentos (listas de cotejo) incluyen ítems o preguntas para guiar la lectura crítica y analizar la validez interna, los resultados y la validez externa de los estudios.

En este artículo describiremos guías de lectura crítica para los diferentes tipos de preguntas y para la mayoría de los diseños observacionales de investigación. Si bien ya habíamos aborda- 
do esta cuestión en EVIDENCIA $A^{\S}$ sin embargo y aunque muchas de las preguntas en la investigación médica provienen de los estudios observacionales ${ }^{6}$, no le habíamos dado aun el merecido tratamiento (a excepción de las series de $\operatorname{casos}^{7}$ ).

Gran parte de la investigación sobre causas de enfermedades depende de estudios de cohortes, caso-control, o transversales, por lo que los estudios observacionales tienen también un papel en la investigación sobre los beneficios y daños de las intervenciones médicas ${ }^{8}$.

Los ensayos aleatorios no pueden contestar todas las preguntas importantes acerca de una intervención determinada. Por ejemplo, los estudios observacionales son más adecuados para detectar efectos adversos de los tratamientos raros o tardíos y probablemente reflejen mejor la práctica médica cotidiana ${ }^{9}$

La credibilidad de la investigación depende de una evaluación crítica de las fortalezas y las debilidades en el diseño del estudio, y de las de la ejecución y el análisis. La información transparente también es necesaria para juzgar si los resultados pueden ser incluidos en revisiones sistemáticas ${ }^{10,11}$.

Sin embargo, la información publicada en la investigación observacional importante es a menudo incompleta o confusa. Un análisis de estudios epidemiológicos publicados en revistas médicas generales y especializadas encontró que la justificación de la elección de las variables de confusión* potenciales a menudo no se reporta ${ }^{12}$.

Por ejemplo, sólo unos pocos informes de estudios caso-control en psiquiatría explican los métodos utilizados para identificar los casos y los controles ${ }^{13}$; en una encuesta, $35 \%$ de los estudios longitudinales en la investigación de accidentes cerebrovasculares, no especificaron los criterios de elegibilidad ${ }^{14}$.
Una red de metodólogos, investigadores y editores de revistas elaboró recomendaciones para la presentación de reportes de investigación observacional.

Esta iniciativa o declaración que desarrolló recomendaciones precisas sobre lo que debe incluirse en un informe completo de un estudio observacional se denominó "Fortalecimiento de la presentación de Reportes de los Estudios Observacionales en Epidemiología" (Strengthening the Reporting of Observational Studies in Epidemiology: STROBE) ${ }^{15}$.

Sin embargo debe destacarse que la declaración STROBE no se desarrolló como una herramienta para evaluar la calidad de la investigación observacional. Tales instrumentos han sido desarrollados por otros grupos y fueron objeto de una revisión sistemática reciente ${ }^{16}$.

El contenido de cada herramienta identificada fue valorado para dominios potencialmente relacionados con sesgos establecidos en la declaración STROBE. Se examinaron 86 herramientas, comprendiendo 41 listas de verificación simples, 12 listas de verificación con juicios sumarios adicionales y 33 escalas. Sólo un tercio fue diseñado para la evaluación crítica.

La mayoría de las herramientas incluían ítems sobre los métodos de selección (92\%) sobre la medición de variables de estudio $(86 \%)$ sobre fuentes de sesgo de diseño específicas (86\%) sobre el control de potenciales confundidores $(78 \%)$ y sobre el uso apropiado de las estadísticas $(78 \%)$. Sin embargo, solamente el $4 \%$ valoró los potenciales conflicto de interés.

La distribución y ponderación de dominios entre herramientas fueron variables e inconsistentes, aunque los principales dominios presentes en las herramientas de valoración de riesgo de sesgo en estudios observacionales pueden verse en la tabla 1.

Tabla 1: principales dominios presentes en herramientas de valoración del riesgo de sesgo en estudios observacionales

\begin{tabular}{l|l|l}
\multicolumn{2}{c|}{ Dominios } & \\
\hline \multirow{3}{*}{ Mayores } & Métodos de selección de participantes & Apropiada fuente poblacional (casos, controles y cohortes) y criterios de inclusión o exclusión. \\
\cline { 2 - 3 } & Métodos de medición de exposición y resultados & Apropiados métodos de medición de exposición y/o resultados. \\
\cline { 2 - 3 } & Métodos de control de confundidores & Diseño y/o métodos analíticos apropiados. \\
\hline \multirow{3}{*}{ Menores } & Sesgo diseño-específico (excluyendo confundidores) & Apropiados métodos para manejar sesgos como el de recuerdo, del entrevistador, pérdidas \\
\cline { 2 - 3 } & Métodos estadísticos (excluyendo el control de confundidores) & $\begin{array}{l}\text { sesgadas de seguimiento o ceguera. } \\
\text { Apropiados métodos estadísticos para el análisis primario de efectos. }\end{array}$ \\
\hline
\end{tabular}

Concluyó que las herramientas deben ser rigurosamente desarrolladas basadas en evidencias, y ser válidas, seguras y fáciles de usar; habiendo necesidad de llegar a acuerdos respecto de elementos críticos para claves el riesgo de sesgo en epidemiología observacional y desarrollar herramientas de evaluación apropiadas.
Expuestas las limitaciones presentaremos a modo de guía, la descripción de los principales tipos de diseño observacional (resumidos en la tabla 2) y algunas listas de cotejo seleccionadas como las más útiles para la evaluación crítica de los estudios observacionales, frecuentemente usados en epidemiología o en la elaboración de revisiones sistemáticas de la evidencia científica. 
Tabla 2: ventajas y desventajas de los estudios analíticos observacionales en epidemiología.

\begin{tabular}{|c|c|c|}
\hline Estudio & Ventajas & Desventajas \\
\hline $\begin{array}{l}\text { Reportes de } \\
\text { caso y series } \\
\text { de casos }\end{array}$ & $\begin{array}{l}\text { - Sencillos, baratos y fáciles de efectuar. } \\
\text { - Pueden comunicar rápidamente sus resultados. } \\
\text { - Buenos generadores de hipótesis. }\end{array}$ & $\begin{array}{l}\text { - No reflejan a la población general ya que pueden basarse en subpoblaciones de } \\
\text { características excepcioneles. } \\
\text { - Sujetos a sesgos (de referencia*, de diagnóstico*, etc.). }\end{array}$ \\
\hline $\begin{array}{l}\text { Corte } \\
\text { transversal }\end{array}$ & $\begin{array}{l}\text { - Determinan la prevalencia de un problema en un momento dado } \\
\text { - Exploran la asociación de diferentes eventos de interés } \\
\text { - Generan hipótesis de asociación causal } \\
\text { - Rápidos y económicos }\end{array}$ & $\begin{array}{l}\text { - La información sobre factores de riesgo y eventos es simultánea, lo que impide interpretar } \\
\text { la relación causa-efecto. } \\
\text { - Una población en estudio no representativa puede generar inferencias erróneas sobre una } \\
\text { población mayor. } \\
\text { - Son susceptibles de todo tipo de sesgos (selección`, información* y confundidores) } \\
\text { difíciles de controlar. }\end{array}$ \\
\hline Caso-control & $\begin{array}{l}\text { - Eficientes (costo y rapidez) } \\
\text { - Establecen posibles causas a través de la asociación entre factores } \\
\text { asociados y el evento de interés. } \\
\text { - Muy útiles para el estudio de eventos de baja prevalencia e } \\
\text { incidencia. }\end{array}$ & $\begin{array}{l}\text { - Susceptibles a muchos sesgos } \\
\text { - Falta de adecuación de la información a analizar (p.ej. revision de historias clínicas). } \\
\text { - Recuerdo selectivo y diferente a determinada exposición entre casos y controles (sesgo de } \\
\text { recuerdo*). } \\
\text { - Problemas de definición de casos y selección de una adecuada población control. } \\
\text { - Distribución desequilibrada de muchas variables que actúen como confundidores. }\end{array}$ \\
\hline Cohortes & $\begin{array}{l}\text { - Establece secuencia temporal } \\
\text { - Mide incidencia } \\
\text { - Estima riesgos absolutos } \\
\text { - Mejor control en la selección de la población en estudio } \\
\text { - Identificación confiable de los eventos en estudio } \\
\text { - Puede evaluar exposiciones poco frecuentes }\end{array}$ & $\begin{array}{l}\text { - Insume costo y tiempo. } \\
\text { - Pérdida de seguimiento en los grupos. } \\
\text { - Sesgo de vigilancia* (búsqueda más exhaustiva en los expuestos). } \\
\text { - Distribución no balanceada de variables confundidoras. } \\
\text { - No es práctico para evaluar eventos poco prevalentes. }\end{array}$ \\
\hline Ecológico & $\begin{array}{l}\text { - Económicos y relativamente fáciles de realizar. } \\
\text { - Muy buena fuente de ideas (hipótesis) para otras investigaciones. } \\
\text { - Permiten la utilización de bases de datos y registros rutinarios }\end{array}$ & $\begin{array}{l}\text { - Debido a que estudian poblaciones enteras, las observaciones realizadas no pueden } \\
\text { extrapolarse al caso del paciente individual ("falacia ecológica"). } \\
\text { - No pueden ser utilizados para poner a prueba estas hipótesis, debido a que no utilizan grupo } \\
\text { control. } \\
\text { - Son particularmente susceptibles a presentar sesgos y confundidores* sin posibilidad de ajuste }\end{array}$ \\
\hline
\end{tabular}

\section{Los reportes de casos y las series de casos}

Los reportes de caso están entre los estudios observacionales más básicos y comunes. Describen la experiencia de los médicos en una presentación o diagnóstico únicos. Los casos múltiples similares constituyen una serie de casos, incrementando la utilidad de la información. La fuerza de los reportes y las series de casos radica en su capacidad única de servir de un sistema de alerta avanzada, permitiendo comunicar rápidamente las nuevas enfermedades, los tratamientos exitosos o fallidos y las complicaciones de un tratamiento o procedimiento. Estos estudios son generalmente económicos y se llevan a cabo rápidamente. Por supuesto, los reportes y series de casos no reflejan la población general ya que frecuentemente se concentran en presentaciones excepcionales. Pueden ser fuertemente influenciadas por el sesgo de referencia de los pacientes más serios a ciertos centros. Sin embargo, son buenos generadores hipótesis y de estudios subsiguientes. Si bien todavía no ha sido desarrollada una herramienta de lectura crítica de las series de caso, sugerimos utilizar el instrumento modificado que se presenta en la tabla 3. Ver más adelante.

\section{El estudio de corte transversal (cross-sectional)}

Se definen como transversales aquellos en los que las mediciones de las variables de interés se realizan en un mismo momento para las unidades de estudio incluidas ${ }^{17,19}$. Habitualmente, estos estudios tienen la finalidad de estimar la frecuencia (prevalencia) de un estado o situación, o una medida de asociación (odds ratio*) entre una exposición y un resultado, aunque no se disponga de seguimientos temporales ${ }^{19}$. En un estudio de corte transversal, una población es observa- da a un determinado momento para establecer características de salud o enfermedad y sus factores asociados (ver figura 1).

Figura 1: esquema general de un estudio de corte transversal.

$$
\text { Corte Transversal }
$$

POBLACION

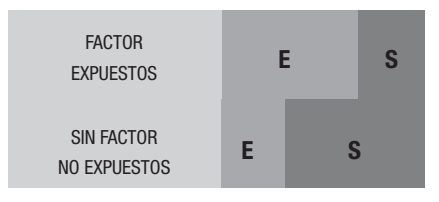

PRESENTE

E: enfermo S: sano

La fortaleza radica en su utilidad para determinar la carga de la enfermedad en una población y para generar hipótesis de las relaciones de exposición a factores posiblemente relacionados y a desenlaces (p. ej. enfermedades). Son particularmente útiles estudiando las enfermedades de larga duración y son relativamente económicos. Sus debilidades incluyen su incapacidad de describir suficientemente enfermedades raras ya que requerirían poblaciones de estudio enormes, lo que es a menudo poco práctico. Las enfermedades de duración breve, como infecciones del tracto respiratorio superior, tampoco son bien evaluadas por este tipo de estudio porque las exposiciones y los resultados de la enfermedad son medidos al mismo tiempo, y las asociaciones de causa-efecto parecerán débiles, aunque sean fuertes. Aunque un estudio de corte transversal es visto como una fotografía en un instante a tiempo, en algunos casos como las encuestas, podrían tardar meses, incluso años, en terminar. En ese tiempo, las exposi- 
15. El análisis estadístico fue determinado desde el inicio del estudio (no post-hoc).

16. Se especifican las pruebas estadísticas utilizadas y son adecuadas.

17. Se trataron correctamente las pérdidas de participantes, datos perdidos u otros efectos del diseño de la muestra (diferentes probabilidades de selección) 0 de la exclusión de casos para algunos análisis.

18. Se tuvieron en cuenta los principales elementos de confusión posibles en el diseño y en el análisis. En el diseño deberían incorporarse variables teóricamente asociadas o determinantes del problema estudiado. En el análisis, la estimación del resultado principal debería estratificarse 0 ajustarse por esas variables.

En resumen, el análisis es adecuado y se minimiza la posibilidad de confusión.

19. Se incluyen resultados de todos los participantes y se indica el número de datos no disponibles.

20. Se presentan los resultados planteados en los objetivos y todos los de interés, de manera clara y

f. Resultados comprensible.

21. Se presentan medidas brutas y ajustadas, indicando las variables por las que se ajustan los resultados y justificando cuáles se incluyeron (o no) en el análisis.

22. Se presentan estimaciones de la significación estadística de las diferencias entre grupos (p. ej. valores de p) 0 de la precisión de los resultados (p. ej. intervalos de confianza).

En resumen, los resultados están bien descritos, son útiles y precisos.

23. Las conclusiones dan respuesta a los objetivos del estudio

g. Conclusiones

24. Las conclusiones presentadas se basan en los resultados obtenidos

25. Los resultados de este estudio pueden extrapolarse a la población de interés de la presente revisión.

Analizar similitudes y diferencias de ambas poblaciones (la del estudio y la de interés del lector) considerando

el contexto espacial y temporal (p. ej. la prevalencia de la exposición) los criterios de inclusión, la definición y la

medición de la exposición/intervención y el resultado, el nivel de confianza de las estimaciones, etc.

25. Los resultados de este estudio pueden extrapolarse a la población de interés de la presente revisión.

Analizar similitudes y diferencias de ambas poblaciones (la del estudio y la de interés del lector) considerando

el contexto espacial y temporal (p. ej. la prevalencia de la exposición) los criterios de inclusión, la definición y la

medición de la exposición/intervención y el resultado, el nivel de confianza de las estimaciones, etc.

26. La discusión explica eventuales extrapolaciones y considera las implicancias de la aplicación de los

resultados, los beneficios, la seguridad y los costos de su aplicación.

En resumen, los resultados del estudio son generalizables a la población y contexto en que interesa aplicarlos.

\begin{tabular}{l|l}
\hline h. Conflicto de & 27. Se menciona la fuente de financiación del estudio o los autores declaran la existencia 0 ausencia de
\end{tabular}

intereses

conflictos de intereses.

En resumen, los conflictos de intereses no condicionan los resultados ni las conclusiones del estudio.

i. seguimiento

28.Se indica el periodo de seguimiento.

(sólo aplicable para 29.Se produjeron pérdidas (anotar el número).

\begin{tabular}{l|l} 
series de casos y & $30 . S e$ indican las características de las pérdidas.
\end{tabular}

\begin{tabular}{l|l} 
estudios & 31. Las pérdidas fueron similares en todos los grupos.
\end{tabular}

antes-despues) $\quad$ En resumen, seguimiento es adecuado.

\begin{tabular}{l|l|c}
\hline VALORACIÓN SUMARIA: & Media & Baja \\
\hline $\begin{array}{l}\text { De la validez interna. Considerar las dimensiones b-e para definir si el diseño del estudio permite minimizar los sesgos y el efecto de } \\
\text { confusión }\end{array}$ & & \\
\hline $\begin{array}{l}\text { De la validez externa. Considerar las preguntas } 25 \text { y } 26 \text { para definir si los resultados son generalizables a la población y contexto en } \\
\text { que interesa aplicarlos }\end{array}$ & & \\
\hline Global de la calidad del estudio. La calidad de la evidencia aportada por el estudio es ${ }^{b}$ & & \\
\hline
\end{tabular}

MB: muy bien. B: bien. R: regular. M: mal. NI: no informa. NA: no aplicable.

aSi bien la definición de confusión implica una relación causal, se utiliza este término para indicar la necesidad de tener en cuenta otras variables que

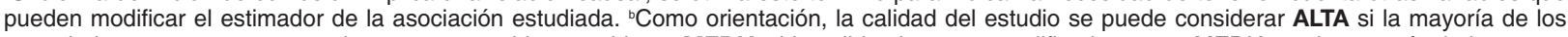
enunciados resumen se responden como «muy bien» o «bien»; MEDIA si la validez interna es calificada como «MEDIA», o la mayoría de los enunciados resumen se responden como «bien» o «regular», y BAJA si la validez interna es calificada como «BAJA», o la mayoría de los enunciados de resumen se responden como «regular» 0 «mal».

Fuente : adaptado de: Berra S y col. Instrumento para la lectura crítica y la evaluación de estudios epidemiológicos transversales. Gac Sanit [revista en la Internet]. 2008 Oct [citado 2010 Mar 24] ; 22(5): 492-497.

Disponible en: http://scielo.isciii.es/scielo.php?script=sci_arttext\&pid=S0213-91112008000500015\&Ing=es y de Scottish Intercollegiate Guidelines Group. A guideline developers' handbook. Edinburgh: SIGN; 2001. Publication N.o 50. 
En la próxima entrega de EVIDENCIA abordaremos aspectos de la lectura crítica de otros tres diseños observacionales: los estudios de casos y controles, los estudios de cohortes y los estudios ecológicos o de correlación.

Recibido el 01/08/10 y aceptado el 27/11/10.

\section{Referencias}

1. Last J, editor. A dictionary of epidemiology. 4th ed. Oxford: Oxford University Press \& International Epidemiological Association; 2001.

2. Porta Serra M. La observación clínica y el razonamiento epidemiológico. Med Clin (Barc). 1986;87:816-9.

3. Luepker RV. Observational studies in clinical research. J Lab Clin Med. 2005 Jul;146(1):9-12.

4. Alvarez-Dardet C y col. Tipos de estudios. Med Clin (Barc). 1987;89:296-301

5. Taubes G. Epidemiology faces its limits. Science. 1995;269:164-9.

6. Glasziou P y col. (2004) Assessing the quality of research. BMJ 328: 39-41

7. errasa S, Caccavo T, Ferraris J, y col. El reporte de un caso y las series de casos. Evid. actual. práct. ambul; 10(1): 19-22, ene-feb.2007.

Disponible: http://www.foroaps.org/files/guia\%20de\%20serie\%20de\%20casos.pdf

8. Black N (1996) Why we need observational studies to evaluate the effectiveness of health care. BMJ 312: 1215-1218.

9. Papanikolaou P y col. (2006) Comparison of evidence on harms of medical interventions in randomized and nonrandomized studies. CMAJ 174: 635-641.

10. Jüni P y col. (2001) Systematic reviews in health care: Assessing the quality of controlled clinical trials. BMJ 323: 42-46

11. Egger M y col. (1998) Spurious precision? Meta-analysis of observational studies. BMJ 316: 140-144.

12. Pocock $S$ y col. (2004) Issues in the reporting of epidemiological studies: a survey of recent practice. BMJ 329: 883.

13. Lee W y col. (2007) Bias in psychiatric case-control studies: literature survey. Br J Psychiatry 190: 204-209.

14. Tooth L y col. (2005) Quality of reporting of observational longitudinal research. Am J Epidemiol 161: 280-288.

15. von Elm E y col. The Strengthening the Reporting of Observational Studies in Epidemiology (STROBE) statement: guidelines for reporting observational studies. Lancet. 2007 Oct 20;370(9596):1453-7.

16. Sanderson $\mathrm{S}$ y col. Tools for assessing quality and susceptibility to bias in observational studies in epidemiology: a systematic review and annotated bibliography. Int $\mathrm{J}$ Epidemiol. 2007 Jun;36(3):666-76.

17. Last J, editor. A dictionary of epidemiology. 4th ed. New York: Oxford University Press; 2001

18. Berra S y col. Instrumento para la lectura crítica y la evaluación de estudios epidemiológicos transversales. Gaceta Sanitaria. 2008;22:492-7.

19. Scottish Intercollegiate Guidelines Group. A guideline developers' handbook. Edinburgh: SIGN; 2001. Publication N.o 50.

20. Silva $L$ y col. A tool for assessing the usefulness of prevalence studies done for surveillance purposes: the example of hypertension. Rev Panam Salud Publica. 2001 Sep;10(3):152-60 